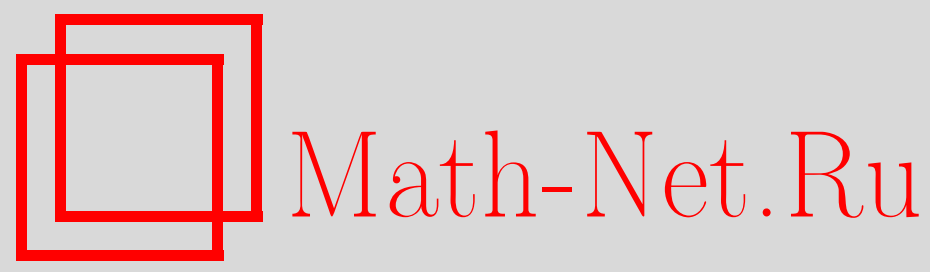

В. В. Ежов, Г. Шмальц, Простое доказательство теоремы Белошапки о параметризации группы автоморфизмов CR-многообразий, Матем. заметки, 1997, том 61, выпуск 6, 939-942

DOI: https://doi.org/10.4213/mzm1578

Использование Общероссийского математического портала Math-Net.Ru подразумевает, что вы прочитали и согласны с пользовательским соглашением http://www . mathnet.ru/rus/agreement

Параметры загрузки:

IP : 18.234 .156 .22

26 апреля 2023 г., 13:33:02

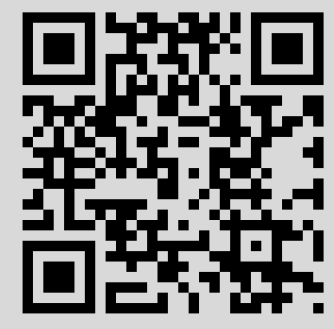




\section{ПРОСТОЕ ДОКАЗАТЕЛЬСТВО ТЕОРЕМЫ БЕЛОШАПКИ О ПАРАМЕТРИЗАЦИИ ГРУППЫ АВТОМОРФИЗМОВ CR-MНОГООБРАЗИЙ}

\section{В.В. Ежов, Г. Шмальц}

Пусть $M \subset \mathbb{C}^{n+k}$ - вещественно-аналитическое CR-многообразие коразмерности $k$ и CR-размерности $n$. В подходящих координатах $\left(z^{1}, \ldots, z^{n}, w^{1}=u^{1}+i v^{1}, \ldots, w^{k}=\right.$ $\left.u^{k}+i v^{k}\right) M$ записывается уравнением $v=F(z, \bar{z}, u)$, где $F(z, \bar{z}, u)-\mathbb{R}^{k}$-значная вещественно-аналитическая функция, которая может быть представлена в виде

$$
F(z, \bar{z}, u)=\sum_{l=0}^{\infty} F_{l}(z, \bar{z}, u)
$$

где $F_{l}\left(t z, t \bar{z}, t^{2} u\right)=t^{l} F_{l}(z, \bar{z}, u)$. Заменой переменных члены $F_{0}$ и $F_{1}$ исключаются , а член $F_{2}$ приводится к виду $F_{2}=\langle z, z\rangle$, где $\langle\cdot, \cdot\rangle-\mathbb{C}^{k}$-значная эрмитова форма в $\mathbb{C}^{n}$.

Многообразие $M$ назьвается невырожсднным по Леви в точке 0 , если следуюшие условия вьполняются:

1) из $\langle a, z\rangle=0$ для всех $z \in \mathbb{C}^{n}$ следует, что $a=0$;

2) формы $\langle\cdot, \cdot\rangle \varkappa$ линейно независимы.

Через $G$ обозначим группу локальных изотропньх автоморфизмов $M$, т.е. ростков биголоморфных отображений $\varphi$ в 0 таких, что $\varphi(0)=0, \varphi(M) \subset M$. В 1990 году Белошапка доказал следующий результат [1].

ТЕОрема 1. Пусть $M$ - невырохсденное в нуле вещественно-аналитическое CR-многообразие $и \varphi \in G$. Тогда $\varphi=(f, g)$ однозначно определяется своими первыми и вторыми производными в нуле.

ДокАЗАТЕЛЬство. Как в оригинальном доказательстве, пользуясь схемой Мозера [2], вьводим некоторую систему дифференциальных уравнений. Пусть $\varphi(z, w)=$ $\left(z^{*}=f(z, w), w^{*}=g(z, w)\right), \mathrm{a}$

$$
f=\sum_{q=0}^{\infty} f_{q} \quad \text { и } \quad g=\sum_{q=0}^{\infty} g_{q},
$$

где $f_{q}\left(t z, t^{2} w\right)=t^{q} f_{q}(z, w)$ и $g_{q}\left(t z, t^{2} w\right)=t^{q} g_{q}(z, w)$.

Тогда $f_{0}=0, f_{1}=C z, g_{0}=g_{1}=0, g_{2}=\rho w$, где $C \in \operatorname{GL}(n, \mathbb{C})$ и $\rho \in \operatorname{GL}(k, \mathbb{R})$ такие, что $\langle C z, C z\rangle=\rho\langle z, z\rangle$.

Условие $\varphi(M) \subset M$ означает, что

$$
\operatorname{Im} g(z, w)=\langle f(z, w), f(z, w)\rangle+\sum_{l=3}^{\infty} F_{l}(f, \bar{f}, \operatorname{Re} g)
$$

Работа первого автора выполнена при финансовой поддержке Австралийского фонда фундаментальных исследований (ARC). Работа второго автора выполнена при финансовой поддержке Немецкого общества исследований (DFG). 
для

$$
\operatorname{Im} w=\langle z, z\rangle+\sum_{l=3}^{\infty} F_{l}(z, \bar{z}, \operatorname{Re} w) .
$$

Выделяя $\mu$-ю компоненту, получаем

$$
\left.\operatorname{Re}\left(i g_{\mu}+2\left\langle f_{\mu-1}, C z\right\rangle\right)\right|_{v=\langle z, z\rangle}=F_{\mu}(z, \bar{z}, u)-F_{\mu}(C z, \bar{C} \bar{z}, \rho u)+\cdots,
$$

где многоточие обозначает выражения, зависящие только от $F_{\nu}, g_{\nu}, f_{\nu-1}, \nu<\mu$. Эта рекуррентная формула однозначно определяет $g_{\mu}, f_{\mu-1}$, если младшие компоненты уже известны и соответствующая однородная система

$$
\left.\operatorname{Re}\left(i g_{\mu}+2\left\langle f_{\mu-1}, C z\right\rangle\right)\right|_{v=\langle z, z\rangle}=0
$$

имеет только тривиальное решение. При замене $f$ на $C f$ и $g$ на $\rho g$ эта система принимает вид

$$
\left.\operatorname{Re}\left(i g_{\mu}+2\left\langle f_{\mu-1}, z\right\rangle\right)\right|_{v=\langle z, z\rangle}=0 .
$$

Собирая в (1) компоненты степени $p$ по $z$ и степени $q$ по $\bar{z}$, производя элементарные преобразования и обозначая теперь через $f_{p}, g_{p}$ мономы степени $p$ по $z$ в старых $f_{\mu-1}$ и $g_{\mu}$, получаем, что $g_{p}=0$ для $p \geqslant 2, f_{p}=0$ для $p \geqslant 3, \operatorname{Im} g_{0}=0$,

$$
\begin{gathered}
g_{1}=2 i\left\langle z, f_{0}\right\rangle, \\
2 \operatorname{Re}\left\langle f_{1}, z\right\rangle=\operatorname{Re} \Delta g_{0}, \\
\left\langle f_{2}, z\right\rangle=2 i\left\langle z, \Delta f_{0}\right\rangle, \\
\operatorname{Im}\left\langle\Delta f_{1}, z\right\rangle=0, \\
\left\langle z, \Delta^{2} f_{0}\right\rangle=0, \\
\operatorname{Re} \Delta^{3} g_{0}=0,
\end{gathered}
$$

где

$$
\Delta=\sum_{\varkappa=1}^{k}\langle z, z\rangle^{\varkappa} \frac{\partial}{\partial u^{\varkappa}}
$$

(cp. [1]).

При $k>1$ получается система дифференциальных уравнений в частных производных с постоянными коэффициентами относительно $u$ ( $z$ фиксировано). Для решения этой системы Белошапка использовал теорему Паламодова об экспоненциальном представлении решений таких систем [3, с. 289-321]. Однако, следующий простой подход позволяет этого избежать. Из (7) непосредственно вытекает, что $\operatorname{Re} g_{0}$, а также $g_{0}$, является многочленом степени не выше 2 по $u$. Из (3) и (5) заключаем, что

$$
\left\langle\Delta^{2} f_{1}, z\right\rangle=0 \text {. }
$$

Покажем, что из (8) следует линейная зависимость $f_{1}$ от $u$. Так как речь идет о полиномиальных решениях, мы предполагаем, что $f_{1}$ - многочлен по $u$ и линеен по $z$. Преобразование Фурье относительно $u$ от (8) равняется

$$
\sum_{|m|=0}^{M}\left\langle\sum_{\nu=1}^{n} \alpha_{m}^{\nu} z^{\nu}, z\right\rangle(\langle z, z\rangle, \xi)^{2} D^{m} \delta=0
$$


где $\xi$ - двойственная переменная к $u, \delta$ - дельта-функция, $(\cdot, \cdot)$ - стандартное скалярное произведение в $\mathbb{R}^{k}, m=\left(m_{1}, \ldots, m_{k}\right)$ - мультииндексы с $|m|=m_{1}+\cdots+m_{k}$,

$$
D^{m}=\frac{\partial^{|m|}}{\left(\partial u^{1}\right)^{m_{1}} \cdots\left(\partial u^{k}\right)^{m_{k}}},
$$

а $\alpha_{m}^{\nu}$ - постоянные $\mathbb{C}^{n}$-векторы.

Без ограничения общности можно считать, что $M$ - наибольшее число такое, что существует ненулевое $\alpha_{m}^{\nu}$ c $|m|=M$. Тогда $M$ равняется степени многочлена $f_{1}$ по $u$. Среди всех ненулевых $\alpha_{m}^{\nu}$ c $|m|=M$ выбираем те, для которьх $m_{1}$ максимально, среди последних выбираем те, для которых $m_{2}$ максимально, и т.д. Таким образом, приходим к некоторому однозначно определенному ненулевому матричнозначному коэффициенту $\alpha_{\tilde{m}}=\left(\alpha_{\tilde{m}}^{\nu}\right)$. Предположим $M \geqslant 2$. Тогда применим функционал из левой части (9) к следующей $\mathbb{R}^{k}$-значной пробной функции $\psi$. Пусть $r \leqslant k$-максимальное число с $\tilde{m}_{r} \neq 0$. Если $\tilde{m}_{r} \geqslant 2$, то положим $\psi=\psi_{0} \xi_{1}^{\tilde{m}_{1}} \cdots \xi_{r}^{\tilde{m}_{r}-2}$.

Иначе, пусть $s<r$ - максимальное число, для которого $\tilde{m}_{s} \neq 0$. Тогда положим $\psi=\psi_{0} \xi_{1}^{\tilde{m}_{1}} \cdots \xi_{s}^{\tilde{m}_{s}-1}$. Вектор $\psi_{0}$ будет определен позднее.

Согласно выбору $\psi$ получаем

$$
\left(\left\langle\sum_{\nu=1}^{n} \alpha_{\tilde{m}}^{\nu} z^{\nu}, \zeta\right\rangle\left(\langle z, \zeta\rangle^{r}\right)^{2}, \psi_{0}\right)=0
$$

или соответственно

$$
\left(\left\langle\sum_{\nu=1}^{n} \alpha_{\tilde{m}}^{\nu} z^{\nu}, \zeta\right\rangle\langle z, \zeta\rangle^{r}\langle z, \zeta\rangle^{s}, \psi_{0}\right)=0
$$

(мы заменили антиголоморфные $z$-переменные на $\zeta$ ).

Выберем $z_{0} \in \mathbb{C}^{n}$ такое, что

$$
\sum_{\nu=1}^{n} \alpha_{\tilde{m}}^{\nu} z_{0}^{\nu} \neq 0
$$

Тогда согласно 1) существует некоторое $\zeta_{0}$ такое, что

$$
\left\langle\sum_{\nu=1}^{n} \alpha_{\tilde{m}}^{\nu} z_{0}^{\nu}, \zeta_{0}\right\rangle \neq 0
$$

По непрерывности неравенство (12) также верно для $z, \zeta$ в достаточно маленьких окрестностях $z_{0}, \zeta_{0}$. Согласно 2) существуют $z_{1}, \zeta_{1}$, принадлежащие этим окрестностям, такие, что $\langle z, \zeta\rangle^{r}$ и $\langle z, \zeta\rangle^{s}$ не обращаются в 0. Тогда для подходящих $\psi_{0}$ левая часть (11) (соответственно (12)) не обращается в 0. Противоречие. Поэтому предположение $M \geqslant 2$ неверно. Следовательно, многочлен $f_{1}$ линеен по $u$.

Точно так же, из (6) можно вывести, что $f_{0}$ линейно зависит от $u$. С учетом (2) отсюда следует, что $g_{1}$ тоже линеен по $u$.

Запишем $\varphi$ в виде

$$
f=f_{0}+f_{1}+f_{2}+\cdots, \quad g=g_{0}+g_{1}+\cdots,
$$


где многоточия обозначают выражения, которые определяются по $f_{0}, f_{1}, f_{2}, g_{0}, g_{1}$. Из (4) следует, что $f_{2}$ определяется по $f_{0}$, из $(2)$ следует, что $g_{1}$ тоже определяется по $f_{0}$. Согласно (3) и (5) $f_{1}$ определяется по $g_{0}$.

Таким образом,

$$
f=z+a w+\cdots, \quad g=w+r(w, w)+\cdots .
$$

В качестве следствия решения системы (1) Белошапка [4] получил явное описание инфинитезимальных автомор физмов невырожденных квадрик $Q=\{(z, w): \operatorname{Im} w=\langle z, z\rangle\}$. Они оказались голоморфными векторными полями

$$
\chi=\sum_{\nu=1}^{n} \zeta^{\nu} \frac{\partial}{\partial z^{\nu}}+\sum_{\varkappa=1}^{k} \omega^{\varkappa} \frac{\partial}{\partial w^{\varkappa}}
$$

такими, что

$$
\left.\operatorname{Re} \chi(v-\langle z, z\rangle)\right|_{v-\langle z, z\rangle=0}=0 .
$$

Коэффициенты $\zeta=\left(\zeta^{\nu}\right)$ и $\omega=\left(\omega^{\varkappa}\right)$ удовлетворяют системе $(1)$, поэтому они являются квадратичными функциями $\zeta=\zeta_{0}+\zeta_{1}+\zeta_{2}, \omega=\omega_{0}+\omega_{1}$, где

$$
\begin{aligned}
& \zeta_{0}=p+a w, \quad \zeta_{1}=X z+B(w, z), \quad \zeta_{2}=A(z, z), \\
& \omega_{0}=q+s w+r(w, w), \quad \omega_{1}=2 i\langle z, p\rangle+2 i\langle z, a \bar{w}\rangle,
\end{aligned}
$$

$p \in \mathbb{C}^{n}, q \in \mathbb{R}^{k},(X, s) \in \mathrm{GL}(n, \mathbb{C}) \times \mathrm{GL}(k, \mathbb{R})$ удовлетворяют условию $2 \operatorname{Re}\langle X z, z\rangle=s\langle z, z\rangle$ для $z \in \mathbb{C}^{n} ; a$ - линейное отображение из $\mathbb{C}^{k}$ в $\mathbb{C}^{n}$ и $A(z, z)-\mathbb{C}^{n}$-значная симметричная билинейная форма на $\mathbb{C}^{n}$ такие, что

$$
\langle A(z, z), z\rangle=2 i\langle z, a\langle z, z\rangle\rangle \quad \text { для } z \in \mathbb{C}^{n}
$$

$B(w, z)-\mathbb{C}^{n}$-значная билинейная форма на $\mathbb{C}^{k} \otimes \mathbb{C}^{n}$ и $r(w, w)-\mathbb{R}^{k}$-значная симметричная билинейная форма на $\mathbb{R}^{k}$ такие, что

$$
\operatorname{Re}\langle B(u, z), z\rangle=r(u,\langle z, z\rangle), \operatorname{Im}\langle B(\langle z, z\rangle, z), z\rangle=0 \quad \text { для } z \in \mathbb{C}^{n}, \quad u \in \mathbb{R}^{k} .
$$

Университет штата Оклахома

Поступило

Институт математики при Боннском университете

15.05 .96

E-mail: schmalz@antigone.mpg.de

\section{СПИСОК ЦИТИРОВАННОЙ ЛИТЕРАТУРЫ}

1. Белошапка В.К. // Матем. заметки. 1990. Т. 47. №3. С. 17-22. 2. Chern S.S., Moser J. K. // Acta Math. 1974. V. 133. №3-4. P. 219-271. 3. Паламодов В. П. Линейные дифференциальные операторы с постоянными коэффициентами. М.: Наука, 1967. 4. Белошапка В. К. // Матем. сб. 1991. Т. 192. № 2. С. 203-219. 